\title{
Lung Ultrasound in COVID-19: Not Novel, but Necessary
}

\author{
Jane A. Shaw Elizabeth H. Louw Coenraad F.N. Koegelenberg \\ Division of Pulmonology, Department of Medicine, Tygerberg Academic Hospital, Stellenbosch University, \\ Cape Town, South Africa
}

A remarkable aspect of the coronavirus disease 2019 (COVID-19) pandemic is the intensity of the search for novel diagnostic and therapeutic tools. This has rightly been driven by the recognition that COVID-19 is a novel disease, with a multitude of clinical manifestations and some unexpected respiratory phenotypes [1].The overwhelming number of severely ill patients with COVID-19 pneumonia has generated a lot of information. Data has poured out of country after country as the wave of the pandemic passed over them, but unfortunately so far very few practically implementable solutions to the many problems of COVID-19 have emerged from the pool.

Amongst those problems is that of how to acquire accurate lung imaging without exposing staff to an unnecessary risk of infection, or placing an extra logistical and financial burden on the already overwhelmed healthcare system. All patients with COVID-19 illness that warrants admission to a hospital will need lung imaging of some kind. The ideal imaging modality for them will be one that is useful for early triaging of patients into mild, moderate, and severe pneumonia; it will be highly specific for COVID-19, and sensitive in ruling it out. It will also be a modality which allows for frequent repeat examinations so that the clinician can use it to monitor progression or

karger@karger.com

(c) 2020 S. Karger AG, Basel

www.karger.com/res

Karger resolution of the disease. It will be available to the doctor at the bedside of the patient, without the necessity of bringing additional staff and equipment into contaminated areas or moving infectious patients into clean areas. Finally, it should be cheap [2]. Of the imaging modalities we have available, none fulfils all these criteria, but perhaps ultrasound may come close.

Data show that computed tomography (CT) chest is a useful diagnostic tool for COVID-19 with a high sensitivity when applied during an outbreak, at times identifying the disease before the gold standard test becomes positive [3-5]. Moreover, the findings on CT chest show a reasonably predictable course, and the severity of the imaging has been associated with the clinical severity of the disease $[6,7]$. The obvious cons include the fact that CT chest is not inexpensive, and is a scarce resource in many settings. Bringing the patient to the scanner exposes valuable staff members and their equipment to an infectious patient, and the procedure uses precious personal protective equipment. Chest radiography has similar logistical issues and yields far less information than CT chest [8]. Lung ultrasound is by no means a novel diagnostic lungimaging modality. It is a specific point-of-care tool for patients with pneumonia, and has been used for some 

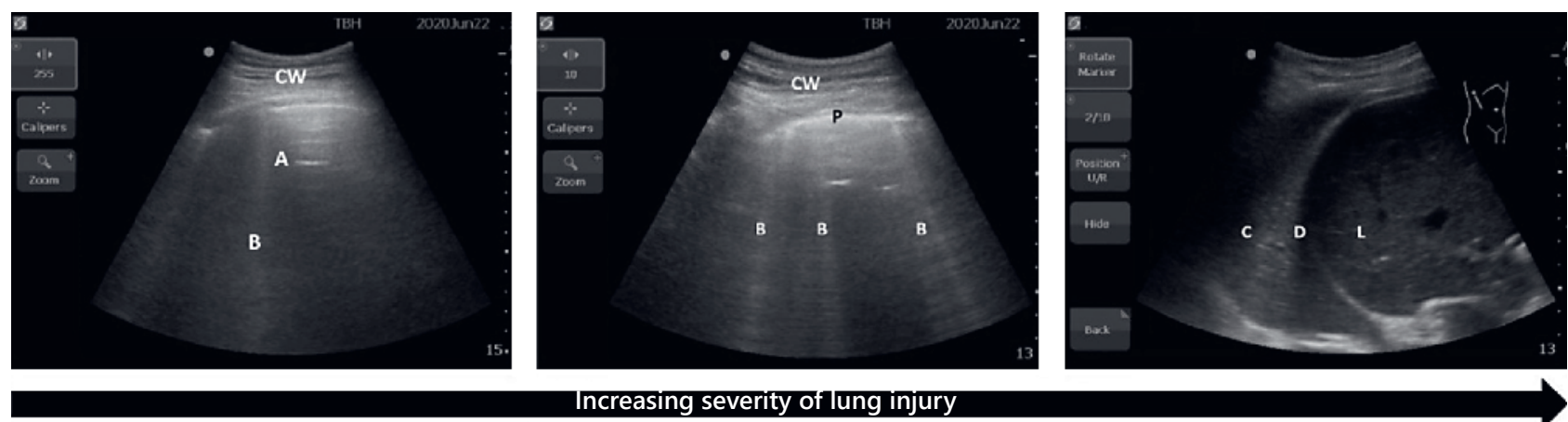

Fig. 1. A simple graphic indicating the potential correlation between lung ultrasound findings and the severity of lung injury. In the left panel, a single B line (B) and the presence of an A line (A) (a normal horizontal reverberation artefact that indicates aerated lung) suggest mild lung injury. This image was obtained from a patient with mild COVID-19 pneumonia. In the middle panel, multiple $\mathrm{B}$ lines $(\mathrm{B})$ and a thickened pleural line $(\mathrm{P})$ indicate more

time now to differentiate patients with dyspnea in the emergency department. Sporadic reports have emerged of its usefulness in distinguishing COVID-19 pneumonia from other causes of respiratory distress in the midst of the outbreak [8-10]. Peng et al. [8] identified the typical features of COVID-19 pneumonia on lung ultrasound as: (1) thickening of the pleural line with pleural line irregularity; (2) B lines in a variety of patterns including focal, multifocal, and confluent; (3) consolidations in a variety of patterns including multifocal small, non-translobar, and translobar with occasional mobile air bronchograms; (4) appearance of A lines during recovery phase; (5) pleural effusions are uncommon. Poggiali et al. [10] performed bedside ultrasound on 12 mildly hypoxic COVID-19 patients in their emergency department and identified a diffuse B-pattern with spared areas in all patients, and posterior subpleural consolidations in 3 of them. These findings correlated strongly to the patients' CT chest images. Lung ultrasound has been used as a tool for monitoring COVID-19 patients from home [11]. In our unit we use it regularly to monitor patients' progress, often as a substitute for examination with a stethoscope or chest radiograph, both of which carry an unacceptably high risk of transmission for the staff members involved [12]. The most common finding in our critically ill patients with COVID-19 pneumonia is B-lines, in keeping with the description from Peng et al. [8] above. B lines are vertical reverberation artefacts on the ultrasound image, generated by abnormalities at the lung-pleura interface, severe lung injury. This image was taken from the same patient as in panel 1 but in a different zone, which emphasizes the need to follow a systematic scoring system which covers all lung zones with each examination. In the right panel, the severest form of COVID-19 lung injury is demonstrated by the presence of consolidated lung (C). D, diaphragm; L, liver; CW, chest wall.

considered to be the pathognomonic finding of the interstitial syndrome [2]. They are thought to be caused by areas of partially deaerated lung, or fluid-filled interlobular septae. Our observation is that the clinical severity of the patient correlates to the degree of lung involvement on ultrasound, including the number of B-lines (Fig. 1). The progress of the patient can thus be tracked by a simplified lung ultrasound score, which is recorded and reported daily. In addition, we find the ultrasound an excellent tool to assess for complications in patients who deteriorate. Lung ultrasound is highly sensitive for the exclusion of pneumothorax, and can identify signs of new infection, heart failure, or pleural effusions [2]. The benefits of a bedside tool that is usable by the physician who is already attending the patient, is easily protected with plastic, easily cleaned, easily moved, and can store images for later perusal are very clear.

In this issue of Respiration, Nouvenne et al. [13] add to the case for ultrasound in COVID-19 with a proof-ofconcept study that assessed the correlation between findings on ultrasound and CT chest. They retrospectively reviewed a small sample of patients admitted with confirmed COVID-19 pneumonia who underwent same-day lung ultrasound and CT chest. Both ultrasound and CT findings were scored according to severity and distribution of abnormalities, and the scores were then tested for correlation. The analysis found a moderately-strong positive correlation between the two which was highly significant $(p<0.001)$, implying that lung ultrasound is not 
inferior to CT chest for the assessment of the severity of lung involvement. Interestingly, the lung ultrasound score also showed a moderately-strong negative correlation to oxygen saturation on room air, reinforcing the conclusion. The only feature which warrants caution is the observation that CT chest scoring correlated positively with mortality in the cohort, while lung ultrasound, disappointingly, did not.

This, as the reports preceding it, is a small study from which we should not make firm conclusions. More research in this area is needed. However, even though the literature is being published at a rate heretofore unimagined, clinicians are still left to make management deci- sions largely without solid evidence on which to base them. Our experience, and these studies, would suggest that lung ultrasound has much to offer to those working on the frontline. Those that advocate for CT chest rather than lung ultrasound are likely to cite a concern over poor ultrasound technique because it is a modality prone to interobserver variability if the user is inexperienced. We would propose that rather than despairing over operator inadequacy, we use the opportunity provided by COVID-19 to train our colleagues in the technique of lung ultrasound - a skill which they will continue to value for the remainder of their careers. COVID-19 calls for novelty, if not in our tools then at least in our attitudes.

\section{References}

1 Gattinoni L, Chiumello D, Caironi P, Busana M, Romitti F, Brazzi L, et al. COVID-19 pneumonia: different respiratory treatments for different phenotypes? Intensive Care Med. 2020 Jun;46(6):1099-102.

2 Shaw JA, von Groote-Bidlingmaier F, Koegelenberg CFN. Transthoracic Ultrasound, in: Palange P, Rhode G, editors: ERS Handbook of Respiratory Medicine. 3rd ed. Sheffield: ERSP; 2019. p. 160-167.

3 Ai T, Yang Z, Hou H, Zhan C, Chen C, Lv W, et al. Correlation of Chest CT and RT-PCR Testing in Coronavirus Disease 2019 (COVID-19) in China: A Report of 1014 Cases. Radiology. 2020 Feb;200642.

4 Feng P, Tianhe Y, Peng S, Shan G, Bo L, Lingli L, et al. Time course of lung changes at chest CT during recovery from coronavirus disease 2019 (COVID-19). Radiology. 2020 Jun; 295(3):715-21.

5 Xie X, Zhong Z, Zhao W, Zheng C, Wang F, Liu J. Chest CT for typical 2019-nCov pneumonia: relationship to negative RT-PCR testing. Radiology. 2020 Feb;200343.
6 Shi H, Han X, Jiang N, Cao Y, Alwalid O, Gu $\mathrm{J}$, et al. Radiological findings from 81 patients with COVID-10 pneumonia in Wuhan, China: a descriptive study. Lancet Infect Dis. 2020 Apr;20(4):425-34. https://doi.org/10.1016/ S1473-3099(20)30086-4.

7 Zhao W, Zhong Z, Xie X, Yu Q, Liu J; Zhao w. Zhong Z, Xie X, Yu Q, Liu J. Relation between chest CT findings and clinical conditions of coronavirus disease (COVID-19) pneumonia: a multicenter study. AJR Am J Roentgenol. 2020 May;214(5):1072-7.

8 Peng QY, Wang XT, Zhang LN; Chinese Critical Care Ultrasound Study Group (CCUSG). Findings of lung ultrasonography of novel corona virus pneumonia during the 2019-2020 epidemic. Intensive Care Med. 2020 May; 46(5):849-50.
9 Farrow R, Becherer-Bailey G, Mantuani D, Nagdev A. Early Multi-organ Point-of-Care Ultrasound Evaluation of Respiratory Distress During SARS-CoV-2 Outbreak: Case Report. Clin Pract Cases Emerg Med. 2020 Apr;4(2):129-133.

10 Poggiali E, Dacrema A, Bastoni D, Tinelli V, Demichele E, Mateo Ramos P, et al. Can Lung US Help Critical Care Clinicians in the Early Diagnosis of Novel Coronavirus (COVID-19) Pneumonia? Radiology. 2020 Jun;295(3):E6.

11 Shokoohi H, Duggan NM, García-de-Casasola Sánchez G, Torres-Arrese M, Tung-Chen Y. Lung ultrasound monitoring in patients with COVID-19 on home isolation. Am J Emerg Med.2020 May;S0735-6757(20)30440$\mathrm{X}$.

12 Buonsenso D, Pata D, Chiaretti A. COVID-19 outbreak: less stethoscope, more ultrasound. Lancet Respir Med. 2020 May;8(5):e27.

13 Nouvenne A, Zani MD, Milanese G, Parise A, Baciarello M, Bignami EG, et al. Lung ultrasound in COVID-19 pneumonia: correlations with chest CT at hospital admission. Respiration. DOI: 10.1159/000509223. 